\title{
LINEAR DIFFERENCE OPERATORS ON PERIODIC FUNCTIONS
}

\section{OTTO PLAAT}

Let $p>0$ and $B$ the Banach space of continuous functions $f: R^{1} \rightarrow R^{1}$ of period $p$, with $\|f\|=\max \{|f(x)| ; 0 \leqq x \leqq p\}$. Let $a \in B, a(x)>0$ for all $x$, and let $t$ be a real number. Define the bounded linear operator $L: B \rightarrow B$ by $L f(x)=f(x+t)-a(x) f(x)$. We shall obtain results concerning the solutions in $B$ of the equation $L f=g$. We say that $L$ is regular if it is one-to-one and onto $B$, and that it is singular otherwise. The limit $\lim _{n \rightarrow \infty}(1 / n) \sum_{k=0}^{n-1} f(x+k t)$, where $f \in B$, will be denoted by $\sigma_{t} f(x)$. If $t / p$ is irrational, the existence of the limit follows from the fact that for every $x$ the sequence $x_{k}=x+k t(\bmod p)$ is uniformly distributed in $[0, p]$, so that $\sigma_{t} f(x)=(1 / p) \int_{0}^{p} f(x) d x$. If $t / p$ is rational, say $q t=r p$, with $q$ and $r$ integers and $q>0$, then $\sigma_{t} f(x)$ $=(1 / q) \sum_{k=0}^{q-1} f(x+k t)$. Note that $\sigma_{t} f(x)=\sigma_{-t} f(x)=\sigma_{t} f(x+t)$.

The case $a(x) \equiv 1$, i.e., the equation

$$
f(x+t)-f(x)=g(x),
$$

was first studied by Euler, whose method was essentially a formal use of Fourier series. The number-theoretic problems which arise via Fourier analysis have been discussed by Wintner [1], who also furnishes an exposition of Euler's work.

The basic result concerning (1) is the following ([2], [3]):

THEOREM 1. Let $g \in B$. There exists $f \in B$ satisfying (1) if and only if $\sum_{k=0}^{n} g(x+k t)$ is bounded (in $x$ and $n$ ).

The application of this result to the general case rests on a trivial reformulation:

THEOREM 2. There exists a positive $b$ in $B$ satisfying $a(x)$ $=b(x+t) / b(x)$ if and only if $\sum_{k=0}^{n} \log a(x+k t)$ is bounded.

An explicit formula for the solutions of (1) will also find an application.

THEOREM 3. The solutions of (1) are given by

$$
f(x)=u(x)-\lim _{n \rightarrow \infty} \frac{1}{n} \sum_{k=0}^{n-1}(n-k) g(x+k t),
$$

Received by the editors December 20, 1965. 
where $u$ is an arbitrary function (in $B$ ) of period $t$.

Proof. Let $f$ be a solution, so that

$$
f(x+(j+1) t)-f(x+j t)=g(x+j t)
$$

for all integers $j$. Sum on $j$ :

$$
f(x+k t)-f(x)=\sum_{j=0}^{k-1} g(x+j t) .
$$

Now sum on $k$ and average:

$$
\begin{aligned}
\frac{1}{n} \sum_{k=1}^{n} f(x+k t)-f(x) & =\frac{1}{n} \sum_{k=1}^{n} \sum_{j=0}^{k-1} g(x+j t) \\
& =\frac{1}{n} \sum_{k=0}^{n-1}(n-k) g(x+k t) .
\end{aligned}
$$

Since the left side converges as $n \rightarrow \infty$, so does the right side, and we have

$$
\sigma_{t} f(x)-f(x)=\lim _{n \rightarrow \infty} \frac{1}{n} \sum_{k=0}^{n-1}(n-k) g(x+k t) .
$$

We shall denote the limit on the right by $\alpha_{t} g(x)$. Noting that $\sigma_{t} f$ has period $t$, and that the solutions of (1) are determined only to within the addition of arbitrary functions (in $B$ ) of period $t$, we may suppose without loss of generality that $\sigma_{t} f=0$. Thus $f=-\alpha_{t} g$, and the addition of an arbitrary $u$ completes the proof. (If $t / p$ is irrational, $u$ is necessarily constant.)

REMARK. Diliberto [3] has shown via a different argument that for any $b \in B$ for which $\sigma_{t} b(x)$ does not vanish, the function

$$
f(x)=-\frac{1}{\sigma_{t} b(x)} \lim _{n \rightarrow \infty} \frac{1}{n} \sum_{k=0}^{n-1} b(x+k t) \sum_{j=0}^{k-1} g(x+j t)
$$

is a solution of (1). Setting $b(x) \equiv 1$ yields the formula $f=-\alpha_{t} g$ obtained above. It is clear that (2) does not, in general, produce all solutions of (1), and that different $b$ 's may produce identical $f$ 's.

Theorem 4. A necessary condition that $L$ is one-to-one is that $\sum_{\mathbf{k}=0}^{n} \log a(x+k t)$ is unbounded. If $t / p$ is irrational, this condition is also sufficient.

Proof. Suppose that the sum in question is bounded, so that by Theorem 2 we may write $a(x)=b(x+t) / b(x)$.

The equation $L f=0$ thus becomes $b(x) f(x+t)-b(x+t) f(x)=0$. 
Since $f=b$ is a nontrivial solution, $L$ is not one-to-one.

Now let $t / p$ be irrational. If $L$ is not one-to-one, there exists $f \neq 0$ such that $f(x+t)=a(x) f(x)$. We show that $f(x)$ cannot vanish. For any $x_{0}, k$,

$$
f\left(x_{0}+(k+1) t\right)=a\left(x_{0}+k t\right) f\left(x_{0}+k t\right),
$$

so that, by elimination,

$$
f\left(x_{0}+n t\right)=f\left(x_{0}\right) \prod_{k=0}^{n-1} a\left(x_{0}+k t\right)
$$

for all $n$. If $f\left(x_{0}\right)=0$, it follows that $f\left(x_{0}+n t\right)=0$ for all $n$. Since the sequence $x_{0}+n t(\bmod p)$ is dense in $[0, p]$ and $f$ is continuous, it follows that $f=0$, a contradiction. Hence there exists a positive $f$ such that $a(x)=f(x+t) / f(x)$, and the conclusion now follows from Theorem 2.

REMARK. A sufficient condition in the rational case $q t=r p$ is easily seen to be that the sum is unbounded on every interval-in other words, that $\left\{x ; \sum_{\substack{a-1 \\ k=0}} \log a(x+k t) \neq 0\right\}$ is dense.

The following is a generalization of Theorems 1 and 3 .

Theorem 5. Let $\sum_{k=0}^{n} \log a(x+k t)$ be bounded. Then the equation $L f$ $=g$ has a solution if and only if $\sum_{k=0}^{n} \exp \left[\alpha_{t} \log a(x+(k+1) t)\right] g(x+k t)$ is bounded. The solutions are given by

$$
f(x)=\exp \left[-\alpha_{t} \log a(x)\right]\left[u(x)-\alpha_{\ell}\left[\exp \left[\alpha_{\iota} \log a(x+t)\right] g(x)\right]\right],
$$

where $u$ is an arbitrary function (in $B$ ) of period $t$.

Proof. By Theorems 1 and 3, we may set

$$
c(x)=\exp \left[\alpha_{t} \log a(x)\right]
$$

so that $a(x)=c(x) / c(x+t)$. The equation $L f=g$ thus becomes

$$
c(x+t) f(x+t)-c(x) f(x)=c(x+t) g(x) .
$$

It is therefore solvable if and only if $\sum_{k=0}^{n} c(x+(k+1) t) g(x+k t)$ is bounded, which is the stated condition. Referring again to Theorem 3 , we find that $-(1 / c(x)) \alpha_{t}[c(x+t) g(x)]$ is a solution, to which may be added an arbitrary solution of $L f=0$. The solutions of the latter are of the form $u(x) / c(x)$. This concludes the proof.

REMARK. The content of Theorem 5 is clarified by noting that the hypothesis implies the factorization $L=Q R S$, where

$Q f(x)=(1 / c(x+t)) f(x), \quad R f(x)=f(x+t)-f(x), \quad S f(x)=c(x) f(x)$. 
Theorem 6. If $L$ is onto $B$, it is one-to-one.

Proof. Consider first the rational case $q t=r p$. If $L$ is onto, there is an $f$ satisfying $f(x+t)-a(x) f(x)=1$. Translating by $k t, k=1$, $2, \cdots, q-1$, we find by elimination that

$$
\left[1-\prod_{k=0}^{q-1} a(x+k t)\right] f(x)=\sum_{n=0}^{q-1} \prod_{k=n+1}^{q-1} a(x+k t),
$$

so that $\prod_{\substack{k-1 \\ k=0}}^{q-1}(x+k t) \neq 1$, all $x$. Hence, $\sum_{k=0}^{q-1} \log a(x+k t)$ does not vanish. The remark following Theorem 4 completes the proof in the rational case.

Now suppose $t / p$ is irrational. If $L$ is not one-to-one, it follows from Theorem 4 that the hypothesis of Theorem 5 is satisfied, and obviously the equation $L f=1$ has no solution.

Theorem 7. A necessary and sufficient condition that $L$ is regular is that $\sigma_{t} \log a(x)$ does not vanish.

Proof. We dispose first of the rational case $q t=r p$. If $L$ is regular, we see, as in the proof of Theorem 6 , that $\sum_{\substack{q-1 \\ k=0}} \log a(x+k t)$ does not vanish. But this is precisely the condition $\sigma_{t} \log a(x) \neq 0$. The sufficiency of the condition follows in the same way; we obtain the formula

$$
L^{-1} g(x)=\frac{\sum_{n=0}^{q-1} g(x+n t) \prod_{k=n+1}^{q-1} a(x+k t)}{1-\prod_{k=0}^{q-1} a(x+k t)} .
$$

We now establish the sufficiency of the condition in the general case. Define operators $T$ and $M$ by $T f(x)=f(x+t), M f(x)$ $=a(x-t) f(x-t)$, so that $L=T(I-M)$. It suffices to show that $I-M$ is regular. We shall, in fact, show that $\left\|M^{n}\right\|<1$ for some (positive or negative) integer $n$. Henceforth let $n$ be a positive integer, and suppose first that $\sigma_{t} \log a(x)<0$. Then

and

$$
M^{n} f(x)=f(x-n t) \prod_{k=1}^{n} a(x-k t), \quad\left\|M^{n}\right\|=\max _{x} \prod_{k=1}^{n} a(x-k t),
$$

$$
\log \left\|M^{n}\right\|=\max _{x} \sum_{k=1}^{n} \log a(x-k t) .
$$

The hypothesis implies that if $n$ is sufficiently large, then $\sum_{k=1}^{n} \log a(x-k t)<0$ for all $x$, and therefore $\left\|M^{n}\right\|<1$ for such $n$. Thus $I-M$ is regular. 
Next, suppose that $\sigma_{t} \log a(x)>0$. Since

$$
M^{-n} f(x)=f(x+n t)\left[\prod_{k=0}^{n-1} a(x+k t)\right]^{-1},
$$

and

$$
\left\|M^{-n}\right\|=\left[\min _{x} \prod_{k=0}^{n-1} a(x+k t)\right]^{-1}
$$

we have that

$$
\log \left\|M^{-n}\right\|=-\min _{x} \sum_{k=0}^{n-1} \log a(x+k t) .
$$

The hypothesis now implies that $\sum_{k=0}^{n-1} \log a(x+k t)>0$ for all $x$, and for $n$ sufficiently large. Hence $\left\|M^{-n}\right\|<1$ for such $n$, and it follows that $M^{-1}-I$ is regular. Since $I-M=M\left(M^{-1}-I\right)$, the sufficiency is proved.

The necessity of the condition in the irrational case will be established by showing that if $\sigma_{t} \log a(x)=0$, there exists a sequence of singular operators which converges to $L$ in norm. Recall that in this case $\sigma_{t} \log a(x)=(1 / p) \int_{0}^{p} \log a(x) d x$. We shall need the following remark: If $t / p$ is irrational and $g(x)$ is a trigonometric polynomial (t.p.) of period $p$ and mean value 0 , then there exists a t.p. $h(x)$ of period $p$ such that $h(x+t)-h(x)=g(x)$. The proof is a simple computation. In fact, if

$$
g(x)=\sum_{0<|k| \leqq N} a_{k} \exp \left(\frac{2 \pi i k x}{p}\right), \quad \bar{a}_{k}=a_{-k},
$$

then

$$
h(x)=\sum_{0<|k| \leqq N} \frac{a_{k}}{\exp (2 \pi i k t / p)-1} \exp (2 \pi i k x / p)
$$

is the desired t.p.

By the Weierstrass approximation theorem, there exists a sequence of t.p.'s $g_{n}(x)$ of period $p$ converging uniformly to $\log a(x)$. Since, by hypothesis, $\int_{0}^{p} \log a(x) d x=0$, we may suppose, modifying the $g_{n}$ if necessary, that $\int_{0}^{p} g_{n}(x) d x=0$. Let $h_{n}(x)$ be a sequence of t.p.'s satisfying $h_{n}(x+t)-h_{n}(x)=g_{n}(x)$. Define the operators

$$
\begin{aligned}
& L_{n} f(x)=f(x+t)-\exp \left[g_{n}(x)\right] f(x), \quad R f(x)=f(x+t)-f(x), \\
& V_{n} f(x)=\exp \left[h_{n}(x+t)\right] f(x), \quad W_{n} f(x)=\exp \left[-h_{n}(x)\right] f(x) .
\end{aligned}
$$


It is readily verified that $L_{n}=V_{n} R W_{n} . R$ is obviously singular, whence $L_{n}$ is singular. But $L_{n}$ converges to $L$ in norm. Hence $L$ is singular.

Corollary. If $\sigma_{t} \log a(x)<0$, then

$$
L^{-1} g(x)=g(x-t)+\sum_{n=1}^{\infty} g(x-(n+1) t) \prod_{k=1}^{n} a(x-(k+1) t),
$$

and if $\sigma_{t} \log a(x)>0$, then

$$
L^{-1} g(x)=-\sum_{n=0}^{\infty}\left[g(x+n t) / \prod_{k=0}^{n} a(x+k t)\right]
$$

the series converging absolutely and uniformly.

Proof. We have the expansion $L^{-1}=(I-M)^{-1} T^{-1}=\sum_{n=0}^{\infty} M^{n} T^{-1}$ in the first case, and

$$
L^{-1}=-\left(I-M^{-1}\right)^{-1} M^{-1} T^{-1}=-\sum_{n=0}^{\infty} M^{-n-1} T^{-1}
$$

in the second. Absolute convergence follows from the norm estimate on $M^{n}\left(M^{-n}\right)$ given in the proof of the theorem. These yield the above expansions for $L^{-1} g(x)$.

\section{REFERENCES}

1. A. Wintner, The linear difference equation of first order for angular variables, Duke J. Math. 12 (1945), 445.

2. W. H. Gottschalk and G. A. Hedlund, Topological dynamics, Colloq. Publ., Vol. 36, p. 135, Amer. Math Soc., Providence, R. I. 1955.

3. S. P. Diliberto, Perturbation theory of periodic surfaces. II, (mimeographed), University of California, 1957; p. 20.

University of SAN Francisco 